\title{
Heme Distortions in Sperm-Whale Carbonmonoxy Myoglobin: Correlations between Rotational Strengths and Heme Distortions in MD-Generated Structures
}

Christoph Kiefl ${ }^{+}$, Narasimha Sreerama ${ }^{+}$, Yi Lu$^{\#}$, Yan Qiu,, John A. Shelnutt ${ }^{\# *}$, and Robert W.

$$
\text { Woody }^{+*}
$$

RECEIVED

AUG $1>2000$

OS T,

${ }^{+}$Department of Biochemistry and Molecular Biology, Colorado State University, Fort Collins, Colorado, 80523, USA.

${ }^{\# B i o m o l e c u l a r}$ Materials and Interfaces Department, Sandia National Laboratories, Albuquerque, NM 87185-1349 (Sandia is a multiprogram laboratory operated by Sandia Corporation, a Lockheed Martin Company, for the United States Department of Energy under Contract DE-AC04-94AL85000), and Department of Chemistry, University of New Mexico, Albuquerque, NM 87131, USA.

Corresponding authors: Robert W. Woody, Phone: (970) 491-6214; Fax: (970) 491-0494, e-mail: rww@lamar.colostate.edu; John A. Shelnutt, Phone: (505) 272-7160; Fax: (505) 272-7077, email: jasheln@unm.edu.

${ }^{+}$Colorado State University

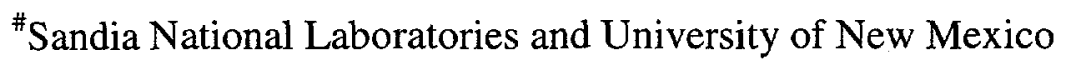

Running Title: Heme Distortions in SW-Myoglobin

Keywords: Sperm-whale myoglobin; heme rotational isomerism; non-planar heme distortions; circular dichroism; rotational strength; normal coordinate structural decomposition. 


\section{DISCLAIMER}

This report was prepared as an account of work sponsored by an agency of the United States Government. Neither the United States Government nor any agency thereof, nor any of their employees, make any warranty, express or implied, or assumes any legal liability or responsibility for the accuracy, completeness, or usefulness of any information, apparatus, product, or process disciosed, or represents that its use would not infringe privately owned rights. Reference herein to any specific commercial product, process, or service by trade name, trademark, manufacturer, or otherwise does not necessarily constitute or imply its endorsement, recommendation, or favoring by the United States Government or any agency thereof. The views and opinions of authors expressed herein do not necessarily state or reflect those of the United States Government or any agency thereof. 


\section{DISCLAIMER}

Portions of this document may be illegible in electronic image products. Images are produced from the best available original document. 


\section{ABSTRACT}

We have investigated the effects of heme rotational isomerism in sperm-whale carbonmonoxymyoglobin using computational techniques. Several molecular dynamics simulations have been performed for the two rotational isomers $\mathrm{A}$ and $\mathrm{B}$, which are related by a $180^{\circ}$ rotation around the $\alpha-\gamma$ axis of the heme, of sperm-whale carbonmonoxy myoglobin in water. Both neutron diffraction and NMR structures were used as starting structures. In the absence of an experimental structure, the structure of isomer B was generated by rotating the heme in the structure of isomer A. Distortions of the heme from planarity were characterized by normal coordinate structural decomposition and by the angle of twist of the pyrrole rings from the heme plane. The heme distortions of the neutron diffraction structure were conserved in the MD trajectories, but in the NMR-based trajectories, where the heme distortions are less well defined, they differ from the original heme deformations. The protein matrix induced similar distortions on the hemes in orientations $\mathrm{A}$ and $\mathrm{B}$. Our results suggest that the binding site prefers a particular macrocycle conformation, and a $180^{\circ}$ rotation of the heme does not significantly alter the protein's preference for this conformation. The intrinsic rotational strengths of the two Soret transitions, separated according to their polarization in the heme plane, show strong correlations with the ruf-deformation and the average twist angle of the pyrrole rings. The total rotational strength, which includes contributions from the chromophores in the protein, shows a weaker correlation with heme distortions. 


\section{INTRODUCTION}

Heme $b$ (protoheme IX) in hemoproteins can exist in two different orientations ${ }^{1}$ that differ by a $180^{\circ}$ rotation of the heme group about the $\alpha-\gamma$ axis (Figure 1), and the two rotational isomers are called isomers A and B. For isomer A, the porphyrin substituents as conventionally numbered (Figure 1) are arranged in a clockwise sense when viewed from the side of the proximal His for globins and other hemoproteins with a single protein ligand, or the C-terminal ligand for bis-liganded hemoproteins (e.g., cytochrome $\mathrm{b}_{5}$ ). For isomer $\mathrm{B}$, the prophyrin substituents are arranged in a counterclockwise sense. Twofold rotation about the $\alpha-\gamma$ axis interchanges the 2- and 4-vinyl groups with the 3-and 1-methyl groups, respectively, and leads to different protein-heme contacts. NMR studies have shown that the equilibrium ratio of $A$ to $B$ form depends on the protein sequence, and on the oxidation state and the ligands of the heme. ${ }^{1}$ In sperm-whale myoglobin, isomer $\mathrm{A}$ is the predominant form. It has been shown that heme distortions can modulate physiologically important properties of hemoproteins. ${ }^{2}$ Heme rotational isomerism could influence these physiologically important properties. Since the protein matrix can induce different distortions in isomers $\mathrm{A}$ and $\mathrm{B}$, the heme in isomer $\mathrm{B}$ may exhibit different functional properties than that in isomer A.

Experimental results initially suggested that isomer B of native sperm-whale myoglobin has a 10-fold higher affinity for $\mathrm{O}_{2}$ than isomer $\mathrm{A}^{3}$. However, subsequent experiments did not show any significant differences in the $\mathrm{O}_{2}$ - and CO-binding of native and reconstituted spermwhale or yellowfin-tuna myoglobin. ${ }^{4-6}$ The differences found in the first experiment were probably due to unrecognized irreversible oxidation of a fraction of heme, which resulted in higher apparent binding constants for $\mathrm{O}_{2} \cdot{ }^{4}$ The $\mathrm{O}_{2}$ affinity is probably modulated mainly by steric and electronic effects depending on the peripheral substituents. ${ }^{7,8}$ However, the Bohr effect in 
Chironomus thummi thummi hemoglobins is controlled by the heme rotational equilibrium. ${ }^{8}$ The major form (isomer A) showed a large Bohr effect, whereas the minor form (isomer B) exhibited little or no Bohr effect. Recent molecular dynamics (MD) simulations ${ }^{9,10}$ of both isomers in sperm-whale myoglobin suggest that the protein matrix induces similar deformations (dihedral angles between two opposite pyrrole rings) of the heme in isomers A and B.

Native sperm-whale myoglobin exhibits heme isomerism, with $5 \%$ of isomer $\mathrm{B}$ at equilibrium. Many high-resolution crystal structures have been reported for myoglobin, but only the A isomer has been observed in crystal structures ${ }^{11}$ and NMR structures. ${ }^{12}$ Freshly reconstituted myoglobin consists of a 50:50 mixture of the two isomers and reaches equilibrium over time. The time required for reaching equilibrium depends on several factors, such as species, pH, temperature, etc. ${ }^{1}$ The Soret $\mathrm{CD}$ spectrum of freshly reconstituted myoglobin is only half as intense as that of the native protein, suggesting that isomer B has a weak, or even a negative, Soret $C D{ }^{5,13}$ Extrapolation of the linear relation between the Soret $\mathrm{CD}$ intensity and the isomeric composition of the reconstituted carbonmonoxy myoglobin, as measured by NMR, gave a $\Delta \varepsilon_{\max }$ of $+90 \mathrm{M}^{-1} \mathrm{~cm}^{-1}$ and $-7 \mathrm{M}^{-1} \mathrm{~cm}^{-1}$, respectively, for isomers $\mathrm{A}$ and $\mathrm{B} .^{14}$

In order to examine the origins of differences in the Soret CD of the two isomers of myoglobin, we have performed MD simulations of sperm-whale myoglobin in both A and B forms. The structure of the B isomer, which is not available experimentally, was generated from the structure of isomer $A$ by rotating the heme about the $\alpha-\gamma$ axis in the heme pocket. The calculated Soret CD spectra of the two isomers are qualitatively similar. The details of the MD analysis and CD calculations have been discussed elsewhere. ${ }^{9,10}$

The origins of the Soret CD lie in the distortions of the heme and the interaction between chromophores of the heme and the protein matrix. ${ }^{15}$ In this paper we present the analysis of the 
protein-induced distortions of the heme in the MD trajectories for isomers $A$ and $B$ using normal-coordinate structural decomposition (NSD) and another simple measure of heme distortion. We correlate the heme deformations with the calculated rotational strengths. The ruf deformation and the average twist angle of the opposite pyrrole rings, which also measures the ruf deformation, show a strong correlation with the two Soret transitions of the heme. The total rotational strength, which includes contributions from other chromophores in the protein, shows a weak correlation.

\section{METHODS}

\section{MD-Simulation.}

Molecular dynamics simulations of the two rotational isomers of sperm-whale carbonmonoxy myoglobin in water have been reported at $300 \mathrm{~K}$ using GROMOS96., ${ }^{9,10}$ The structure of isomer B was generated by a $180^{\circ}$ rotation of the heme about the $\alpha-\gamma$-axis in the protein matrix of isomer $\mathrm{A}$, keeping the heme propionates in the original position of the neutrondiffraction structure (or the NMR structure).

We performed four MD simulations for each isomer, with four different initial velocities, using the neutron-diffraction structure as the starting geometry. This strategy has been recommended for systems with multiple minima, ${ }^{16}$ such as proteins, because several shorter trajectories with different initial velocities sample the conformational space more efficiently than a single simulation of longer duration. One single long trajectory could be trapped in a local energy minimum for a long time and repeatedly explore only a small region of conformational space. These four MD trajectories of isomer A, generated with the neutron-diffraction structure 
and different initial velocities, are referred to as T1A, T2A, T3A, and T4A. The corresponding trajectories of isomer $\mathrm{B}$ are referred to as $\mathrm{T} 1 \mathrm{~B}, \mathrm{~T} 2 \mathrm{~B}, \mathrm{~T} 3 \mathrm{~B}$, and $\mathrm{T} 4 \mathrm{~B}$, respectively. We have also performed MD simulations with two starting structures taken from the ensemble of twelve NMR structures, ${ }^{12}$ structures \#1 and \#8, and these MD trajectories are referred to as $\mathrm{T} 5$ and $\mathrm{T} 6$, respectively, with the isomer indicated by A and B, e.g., T5A for isomer A with NMR structure $\# 1$ as the starting geometry. These MD simulations were performed on two of the original NMR structures instead of using the average of the twelve NMR structures. In this way, we covered more conformational space and additionally could monitor the dependence of MD-generated structures on initial distortions. The trajectories $\mathrm{T} 5$ and $\mathrm{T} 6$ have different starting geometries, whereas trajectories $\mathrm{T} 1$ to $\mathrm{T} 4$ have the same starting geometry but differ in their initial velocities.

\section{Intrinsic Rotational Strength}

Circular dichroism is the differential absorption of left and right circularly polarized light,

$$
\Delta \varepsilon=\varepsilon_{L}-\varepsilon_{R}
$$

and the rotational strength of a transition from the ground state 0 to excited state $a$ is determined by integrating the intensity under a single band in the CD spectrum, according to

$$
\mathrm{R}_{\mathrm{oa}}=\frac{3 \hbar c}{16 \pi^{2}} \frac{10^{3} \ln 10}{N_{A}} \int \frac{\Delta \varepsilon(\lambda)}{\lambda} d \lambda
$$

where $\hbar$ is Planck's constant divided by $2 \pi, c$ is the velocity of light, and $N_{A}$ is Avogadro's number. Rotational strength is usually expressed in Debye-Bohr magnetons $(1 \mathrm{DBM}=0.9273 \mathrm{x}$ $10^{-38}$ cgs units).

The rotational strength can be calculated theoretically from the imaginary part of the 
scalar product of the electric and magnetic transition dipole moments:

$$
\mathrm{R}_{\mathrm{oa}}=\operatorname{Im}\left(<\psi_{\mathrm{o}}\left|\mu_{\mathrm{e}}\right| \psi_{\mathrm{a}}>\bullet<\psi_{\mathrm{a}}\left|\mu_{\mathrm{m}}\right| \psi_{0}>\right)
$$

where $\mu_{e}$ is the electric dipole transition moment, a measure of the linear displacement of charge upon excitation; and $\mu_{m}$ is the magnetic dipole transition moment, a measure of the circular displacement of electron density upon excitation. The superposition of $\mu_{e}$ and $\mu_{m}$ results in a helical displacement of charge, which interacts differently with left- and right- circularly polarized light.

In these calculations we treated the heme as a porphyrin dianion, considering only the $\pi$ electrons, neglecting the methyl and propyl side chains, and including the two vinyl groups. The $\pi$-system in the heme group contained 28 atoms. The transition parameters and intrinsic rotational strength of the Soret transitions were calculated using $\pi$-molecular orbital theory in the Pariser-Parr-Pople (PPP) approximation. ${ }^{17}$ The chromophores of the protein matrix, peptides and aromatic side-chain groups, were included in the calculation following Tinoco's first-order perturbation theory as previously described. ${ }^{9,10}$ The calculations were performed on the heme structures in the MD trajectories at 50-fs intervals.

The Soret CD band has two nearly degenerate components that are polarized in the plane of the heme and perpendicular to each other. The near-degeneracy of the Soret components leads to fluctuations in the relative energies of the $x$ - and $y$-polarized Soret components, precluding a unique correlation using the energy classification. The two Soret components were distinguished according to their polarization in the heme-plane, which gives a good correlation with a measure of heme distortion as described below. Each Soret transition moment was assigned as either $x$ - or $y$-polarized, according to the angle of its projection on the mean plane of the heme, using $\pm 45^{\circ}$ 
lines as boundaries. The $x$-axis passes through the nitrogen atoms of the pyrroles $\mathrm{D}$ and $\mathrm{B}\left(\mathrm{N}_{\mathrm{D}^{---}}\right.$ $\mathrm{N}_{\mathrm{B}}$ ) and the $y$-axis through $A$ and $C$ pyrroles $\left(\mathrm{N}_{\mathrm{A}^{---}} \mathrm{N}_{\mathrm{C}}\right)$ (Figure 1 ).

\section{Normal-Coordinate Structural Analysis}

Selected heme structures were analyzed by normal-coordinate structural decomposition (NSD) as described earlier. ${ }^{18,19}$ Figure 2 illustrates NSD for the heme from the X-ray crystal structure of horse heart cytochrome $c$. In practice, the computational procedure projects out the deformations along all of the 66 normal coordinates of the porphyrin macrocycle, including both in-plane and out-of-plane modes. Typically for hemoprotein crystal and NMR structures, however, the deformations are only large enough to be statistically significant for the lowestfrequency out-of-plane modes. This is because these modes have the smallest restoring forces and thus tend to have the largest deformations from planarity. For porphyrin crystal structures, which are usually refined to much higher resolution than hemoproteins, the lowest-frequency

modes alone do not provide a sufficient description of the structure, ${ }^{18}$ but for the hemoproteins, a description in terms of the deformations of only the lowest-frequency normal modes is adequate statistically. That is, the error in the structure when the contributions from all of the other modes are neglected is less than the positional uncertainty in the X-ray structure.

\section{Twist-Angle ( $\tau)$}

The virtual dihedral angles between the two sets of opposing pyrrole rings can be used to characterize the overall distortion of the heme. These relate the pyrrole rings $A \leftrightarrow C$ and $B \leftrightarrow D$ (see Fig. 1) and are denoted $\tau_{\mathrm{AC}}$ and $\tau_{\mathrm{BD}}$, respectively. These two dihedral angles give a measure of the twist of the pyrrole rings with respect to the heme-plane and are inversely related. Generally, a positive $\mathrm{AC}$ twist $\left(0^{\circ}<\tau_{\mathrm{AC}}<180^{\circ}\right)$ is compensated by a negative $\mathrm{BD}$ twist $\left(-180^{\circ}<\right.$ 
$\tau_{\mathrm{AC}}<0^{\circ}$ ), which is a consequence of the constraints of the heme macrocycle. For some small values of $\tau_{\mathrm{AC}}$ and $\tau_{\mathrm{BD}}$ the inverse relation may not be satisfied, but the heme deformations are also small in such cases.

The average twist angle $(\tau)$ was calculated by averaging the absolute values of the two dihedral angles and retaining the sign of the AC twist angle. The average twist angle shows a strong correlation with the ruffling deformation from the NSD analysis. The opposite signs of $\tau_{\mathrm{AC}}$ and $\tau_{\mathrm{BD}}$ occurs for the pro deformation also, but the average pro deformation is much smaller than the ruf deformation.

\section{RESULTS AND DISCUSSION}

\section{Heme Distortions-Normal Coordinate Structural Analysis}

The major heme deformations that make up the distortion in hemoproteins are ruffling (ruf), saddling (sad) and doming (dom), whereas waving (wav(x), wav(y)) and propellering (pro) deformations tend to be smaller due to their higher vibrational frequencies ${ }^{18,19}$ (Figure 2). These six macrocycle deformations are unique because they correspond to the lowest-frequency out-ofplane vibrational modes of $B_{1 u}, B_{2 u}, A_{2 u}, E_{g}$, and $A_{1 u}$ symmetries (irreducible representations), respectively, of the nominal $D_{4 h}$ molecular symmetry of hemes. Using NSD, the out-of-plane distortion of most heme structures can be well represented in terms of the normal coordinate displacements for these six vibrational modes.

The out-of-plane heme distortions in myoglobin are generally small in comparison with

most other hemoproteins. ${ }^{18}$ For the 14 experimental structures of sperm-whale carbonmonoxymyoglobin obtained by X-ray diffraction (1vxf), neutron diffraction (2mb5), and NMR 
spectroscopy (1myf: 12 structures), the total out-of-plane distortions are generally less than 0.7 $\AA$. The consistent features of the experimental heme structures from all sources are the small, positive doming, $y$-waving, and propellering deformations $(<0.5 \AA)$. All other deformations are also small $(<0.4 \AA)$ and can be either positive or negative, although the wav $(x)$ deformation is typically negative.

The NSD results for the 600-ps averaged structures show that deformations of all crystalbased trajectory structures are largely conserved for isomers A and B and differ only in their amplitudes (Figure 3). This is probably because the trajectories have the same starting structure and differ only in their initial velocities. Further, at least the signs of the initial deformations of the neutron-diffraction heme structure are conserved in all of the averaged structures of the crystal-based trajectories for isomer A, except for dom in T3A. However, the 600-ps averaged heme distortions in trajectories T5 and T6 (Figure 3) are very different from the original heme distortions of NMR-structures \#1 and \#8. This may reflect the fact that heme distortions are not completely characterized by NMR signals, i.e., only the outer C-atoms of the heme are well characterized, whereas the positions of the inner C-atoms of the macrocycle are due to the force field used in the NMR structure determination.

The NSD results show that there are clear differences between the neutron-diffraction (crystal) and NMR-based (solution) heme distortions due to the different starting structures (Figure 3). For the 600-ps averaged heme structures (Table 1), the isomer A distortions for the crystal-based trajectories are mostly dominated by positive saddling, ruffling, $y$-waving, and propellering. The isomer A deformations for the NMR-based trajectories have the same sign, except for ruffling and propellering (in trajectory T5A), which are negative. In isomer B, ruffling and saddling dominate the deformations for the crystal-based trajectories: saddling, $y$ - 
waving and propellering are positive, and ruffling, doming and $x$-waving are negative. These signs are again conserved in isomer $\mathrm{B}$, for T5B and T6B, except for ruffling in trajectory T6B.

The comparison of the heme deformations between the isomers $\mathrm{A}$ and $\mathrm{B}$ requires a careful examination. At first sight, there seems to be a clear difference between A and B in all trajectories, but we have to consider the symmetry operations due to the heme rotation (see relationships below). To see the effects of the rotation, we must first understand the way in which the NSD analysis of heme structures is done and the resulting sign convention for the individual deformations. All hemes are analyzed by first orienting the heme as shown in Figure 1 (isomer A). The asymmetric substitution pattern of protoporphyrin allows the heme to be uniquely positioned and thus the heme conformation to be uniquely expressed by the NSDdetermined normal-coordinate displacements. The uniqueness in the orientation used for NSD leads to the unique sign convention for protoporphyrin deformations. Now consider a purely ruffled heme structure. In the ruf deformation, the pyrrole rings are twisted about the metalnitrogen bonds (Figure 2). This twist can occur in a positive or negative sense relative to the mean plane of the heme. For a symmetrically substituted porphyrin, there is ambiguity in the absolute sign of the ruffling because there is no unique way to orient the heme for the NSD analysis, e.g., the macrocycle could be rotated by $90^{\circ}$ about the $z$-axis or rotated about an inplane symmetry axis, reversing the sign of the ruffling. For unsymmetrical hemes, the sign convention is determined because we always orient the heme as shown for isomer A in Figure 1, prior to the NSD analysis.

Now consider the scenario in which the heme is rotated by $180^{\circ}$ about the $\alpha-\gamma$ axis within the specific protein environment of myoglobin. For simplicity, assume that the protein imposes exactly the same distortion on the macrocycle of the rotated heme. Then detailed 
considerations described below show that the NSD deformations will be influenced as follows:

$$
\begin{gathered}
r u f \rightarrow-r u f \\
\text { sad } \rightarrow \text { sad } \\
d o m \rightarrow-d o m \\
w a v(x) \rightarrow-w a v(y) \\
\text { wav }(y) \rightarrow-w a v(x) \\
\text { pro } \rightarrow \text { pro }
\end{gathered}
$$

As an example of how these relationships are determined, consider a heme-binding site that favors a purely ruffled macrocyclic structure in which the $\alpha$ and $\gamma$ positions are above the mean plane and the $\beta$ and $\delta$ positions are below the plane. If the heme is rotated $180^{\circ}$ about the $\alpha-\gamma$ axis and the heme structure is unchanged, then the $\alpha$ - and $\gamma$-carbons are now below the mean plane. This does not change the NSD results, because the heme would be rotated back to the orientation shown in Figure 1 (isomer A) for analysis. However, if the heme site in the protein prefers the $\alpha$ and $\gamma$ positions to be above the plane, the conformation of the rotated heme is changed. This rotated structure, with the $\alpha$ - and $\gamma$-carbons forced above the plane by the protein, must also be put back into the orientation in Figure 1 (isomer A) for the NSD analysis. However, when reoriented, the $\alpha$ and $\gamma$ positions are now below the plane, thus changing the sign of the ruffling as indicated in the above relationship. Similar arguments apply for the other deformation types.

In light of the relationships associated with rotational isomerism in the heme site, the differences in structure of isomers $A$ and $B$ seen in the 600-ps averaged structures (Figure 3) 
make good sense qualitatively. Comparing isomers A and B in Figure 3, we see that ruffling and doming typically have opposite signs for A and B, in agreement with the expected relationships. Also as expected, the sad and pro deformations have the same signs for isomers A and B. Furthermore, if the magnitude of the $w a v(x)$ deformation is large and $w a v(y)$ is small for the $\mathrm{B}$ isomer, then $\operatorname{wav}(y)$ is large and $\operatorname{wav}(x)$ is small for the A isomer. This is consistent with the switching of $x$ and $y$ in the expected relationships. The relative signs of $\operatorname{wav}(x)$ and $\operatorname{wav}(y)$ do not usually differ between isomers $\mathrm{A}$ and $\mathrm{B}$ because these deformations usually have opposite signs for a heme structure. This insures that the minus sign in the relationships for the wave deformation cancels with the relative sign between the deformations, so all we observe is the switching of the magnitudes. From these NSD results, we must conclude that the binding site prefers a particular macrocycle conformation, and that $180^{\circ}$ rotation of the heme does not greatly alter the protein's preference for this conformation.

The 100-ps time-averaged structures show clear evidence of slow oscillations in the heme deformations (Figure 4). This is best seen in Figure 4a for isomer B (trajectory T1B), which shows how the ruffling, which is first small and positive (0-100 ps), becomes larger (100-200 ps), then becomes negative (200-300 ps), and then continues to become more and more negative over the next $300 \mathrm{ps.} \mathrm{A} \mathrm{similar} \mathrm{oscillation} \mathrm{in} \mathrm{the} \mathrm{ruffling} \mathrm{is} \mathrm{observed} \mathrm{for} \mathrm{isomer} \mathrm{A.} \mathrm{Doming} \mathrm{and}$ saddling also show oscillations, but the amplitudes of the oscillation are smaller than for the softer ruffling deformation. These slow oscillations are less obvious for the NMR-based trajectory T6 (Figure 4b), because reversal of the sign of the deformations is rare in the NMRbased trajectories. Nonetheless, it is clear that in all cases much longer MD calculations are required in order to average over these nanosecond-scale oscillations of the macrocycle structure. It must be emphasized that we are dealing here with changes in average structures, obtained by 
averaging over 2000 frames of the trajectory. Therefore clearcut dynamic interpretations are difficult. However, the nanosecond time scale of these oscillations suggests that they may reflect large-scale motions in the protein structure.

Faster oscillations of the deformations are also observed. Figure 5 shows NSD results over 6.4 ps of an MD trajectory (T2A) for snapshot structures obtained at 50-fs intervals. Ruffling shows both the longest period of oscillation $(\sim 0.5 \mathrm{ps})$ and the largest amplitude. The period puts the frequency in the tens of wavenumbers range, close to the expected vibrational frequency ${ }^{18}$ of the normal mode corresponding to the ruffling vibration of the macrocycle. The regular sinusoidal vibrational motion is however altered by interaction with the changing protein environment, as well as other factors. For the other deformations, inspection of Figure 5 shows that the frequency increases and the amplitude decreases, as expected from values obtained from independent normal coordinate analyses of porphyrin motion. That is, the apparent frequencies increase in the order ruf $<s a d<d o m \sim w a v(x) \sim \operatorname{wav}(y)<\operatorname{pro}$. This frequency order is obtained by Fourier transforms of time-domain data shown in Figure 5 (results not shown). The apparent amplitudes decrease in the same order. This order agrees approximately with the order of the frequencies obtained in the normal coordinate calculations used to obtain the normal coordinate eigenvectors used in the NSD analysis. Exact agreement is not expected because the substituent masses are set to zero to obtain the eigenvectors of the bare macrocycle. These macrocycle motions are mixed with the substituent motions to give the normal coordinates of the protoheme $\mathrm{IX}$, and in-phase and out-of-phase mixing may account for high- and low-frequency peaks in the Fourier transforms.

The average values of the $w a v(y)$, and pro deformations (approximately 0.2 and $0.1 \AA$, respectively) evident from the 1-7 ps trajectory (Figure 5) are in good agreement with the longer 
averages of the $0-100-p s$ and $0-600-p s$ averaged structures for the same trajectory (Figure 4). The positive average value for the ruf deformation may also be evident to the eye from Figure 5a. It is important to note that such excursions in ruffling on a picosecond timescale are much larger than the average ruf deformation and the ruffling seen experimentally.

Transient ruffling on the order of $1 \AA$ is large enough to cause $d$-orbital configuration changes in hemes. Nakamura et $a .^{20}$ observed different $d$-orbital configurations for ruffled and planar low-spin Fe(III) porphyrins, demonstrating that ruffling can induce the $\left(d_{t}^{2}, d_{x y}^{2} \rightarrow d_{x=}^{4} d_{x}^{1}\right)$ transition and thereby influence hemoprotein function. Our MD simulations suggest that even for hemeproteins like myoglobin, which show only small static ruffling deformations, deformations of over $1 \AA$ occur transiently, and thus the $d$-orbital configuration may transiently change as well. In proteins like the c-type cytochromes, for which the average structure is already ruffled by about $1 \AA$, even larger excursion in the ruffling may occur transiently. The associated effects on the $d$-orbital occupations could easily influence the electron-transfer function of cytochromes, possibly even providing an electron gating mechanism.

\section{Heme Distortions-Twist Angle}

The average twist-angle $\tau$ can also be used to characterize the overall heme distortions. This represents the average twist of the pyrrole rings with respect the plane of the heme macrocycle and is strongly correlated with the ruffling (ruf distortion) characterized from the NSD analysis. The strong correlation $(r=0.99)$ between these two measures of heme distortion, for the $0-6.4$ ps trajectory of T2A, is shown in Figure 6. Ruffling is a major type of distortion in heme, shows the largest amplitudes and oscillations (Figure 5), and is particularly important in the $C D$ calculations. While it is desirable to characterize each structure from the MD trajectories using the more sophisticated NSD analysis, it is very computationally intensive to perform the 
analysis on each of the 12000 structures of a 600 -ps trajectory; we have 12 such 600 -ps trajectories. For this reason, we have also used the simple measure of $\tau$ for characterizing the heme distortions from the MD trajectories.

The distribution of the twist angles, $\tau_{\mathrm{AC}}$ and $\tau_{\mathrm{BD}}$, for the crystal- and NMR-based simulations are shown in Figure 7. It is clearly evident from this distribution that the positive twist of one set of pyrroles is accompanied by a negative twist of the other set. The strain of the twist from one set of pyrroles, say $\mathrm{A} \leftrightarrow \mathrm{C}$, on the ring system of the heme macrocycle is relieved by twisting the second set of pyrroles $(B \leftrightarrow D)$ in the opposite direction. The range of twist angles is slightly larger in the NMR-based trajectories $\left( \pm 50^{\circ}\right)$ in comparison with the crystal-based trajectories $\left( \pm 45^{\circ}\right)$. The main reason for this behavior is the flexibility of the NMR structure as compared to the crystal structure. This is partly due to the "invisibility" in NMR of all but the peripheral atoms of the macrocycle, and partly due to the somewhat looser structure of the protein in the NMR structures.

The differences between the twist angle distributions in the two isomers is also evidenced in Figure 7. The two distributions are approximately interchanged between the two isomers. In isomer $\mathrm{A}, \tau_{\mathrm{AC}}$ distribution spans negative values, while $\tau_{\mathrm{BD}}$ spans positive values. The situation is reversed in isomer $\mathrm{B}$, where $\tau_{\mathrm{BD}}$ spans negative values and $\tau_{\mathrm{AC}}$ spans positive values. The two isomers are related by a $180^{\circ}$ rotation about the $\alpha-\gamma$ heme-axis, which interchanges the pyrrole rings $\mathrm{A}$ and $\mathrm{B}$, and $\mathrm{C}$ and $\mathrm{D}$, with respect to the protein matrix. This results in the interchange of the two twist angles, $\tau_{\mathrm{AC}}$ and $\tau_{\mathrm{BD}}$, with respect to the protein matrix, making a positive $\tau_{\mathrm{AC}}$ distribution in isomer $\mathrm{A}$ equivalent to a positive $\tau_{\mathrm{BD}}$ distribution in isomer $\mathrm{B}$. The twist angle distributions point to similar overall heme distortions in both isomers in the protein matrix, 
suggesting that the macrocycle binds to the protein in a unique conformation, confirming results from the NSD analysis.

The twist angle distributions for the NMR-based trajectories have an inverse relation with those for the crystal-based trajectories. A similar feature was also observed in the NSD analysis, where the ruffling distortions for the NMR- and crystal-based average structures have opposite signs. As indicated earlier, the NMR structures are less well defined in comparison with the neutron-diffraction structure, and the starting structures for NMR- and crystal-based MD simulations are different. As described below, the CD spectral calculations of MD trajectories also suggest that the NMR trajectories provide an inferior starting structure. It may be that the NMR structures are just sampling a time regime in which the ruf deformation in particular has a different value. Even in the crystal-based trajectory, the ruf deformation can be positive or negative and large for long times.

\section{Correlations between Rotational Strength and Heme Distortions.}

The Soret band of the heme consists of two transitions that are nearly degenerate, polarized in the plane, and at right angles to each other. A planar heme, which is achiral, has zero intrinsic rotational strength. Deviations from the planarity of the heme introduces chirality in the heme and makes the two transitions distinct, resulting in a net intrinsic rotational strength leading to observable Soret $\mathrm{CD}$. The chiral environment of the protein matrix, and the interactions between the chromophores in the protein matrix and the heme can also contribute to Soret CD. The heme structures generated in the MD simulations deviate from planarity and are expected to have intrinsic rotational strength. The intrinsic rotational strength of the heme was calculated using the PPP approximation and treating the heme $\pi$-electron system as a porphyrin dianion. 
While the two Soret transitions have approximately the same energy, the heme distortions and the asymmetric substitution pattern break the degeneracy of a planar, fourfold symmetrical heme. Our initial efforts to correlate the calculated rotational strengths of the two Soret transitions with measures of nonplanar distortions, such as ruffling and the average twist angle, were based on classification into high-and low-energy components. This scheme gave doublevalued correlations because the near-degeneracy of the Soret components leads to fluctuations in the relative energies of the $x$ - and $y$-polarized Soret components and precludes a unique correlation using the energy classification.

Since the two Soret components are polarized perpendicular to each other, the polarization of the two Soret components is more useful for examining correlations. Each Soret transition moment was assigned as either $x$ - or $y$-polarized according to the angle of its projection on the mean plane of the heme, using $\pm 45^{\circ}$ lines as boundaries. The $x$-axis is taken to be the $\beta-\delta$ axis and the $y$-axis as the $\alpha-\gamma$ axis (Figure 1). The intrinsic rotational strengths of the two Soret transitions were compared with the heme distortions as characterized by the average twist angle $\tau$ for the entire MD trajectory, and with the ruffling deformation, which is strongly correlated to $\tau$, for a short 6.4 ps trajectory.

Figure 8 shows how the intrinsic rotational strengths of the heme Soret transitions depend on the average twist angle. For both isomers $\mathrm{A}$ and $\mathrm{B}$, those transitions polarized predominantly along the $y$-axis ( $\alpha-\gamma$ axis) have positive rotational strengths for positive $\tau$ and negative rotational strengths for negative $\tau$. The rotational strengths of transitions polarized along the $x$ axis ( $\beta-\delta$ axis) show an anticorrelation with $\tau$. There is a substantial scatter, as shown by the breadth of the distributions, but the correlation coefficients of $c a . \pm 0.85$ indicate that the average twist angle is a good predictor of the sign and approximate magnitude of the intrinsic heme 
rotational strengths.

As expected from the strong correlation $(r=0.99$; Figure 6$)$ between the ruffling and the average twist angle, the intrinsic rotational strength also shows a good correlation with the ruffling distortion $(r= \pm 0.89)$. The slightly higher correlation coefficient for the ruffling with rotational strength in comparison with the average twist angle $(r \approx \pm 0.85)$ is due to the use of a shorter trajectory (6.4 ps). Correlation with the other distortion modes (sad, dom, pro and wav) is weak. In an unsubstituted porphyrin, the only distortion modes that are chiral are ruffling and propellering. It is therefore clear why ruffling should correlate well with the intrinsic rotational strength of heme transitions. The lack of a good correlation with the propeller distortion may result from the low amplitude $(0.1 \AA)$ of this high-frequency motion.

Figure 8 shows that isomers $\mathrm{A}$ and $\mathrm{B}$ give similar correlations between intrinsic Soret rotational strengths and average twist angle. The range of twist angles in isomer B is somewhat larger than that for isomer $\mathrm{A}$, and so there are more extreme values of $\mathrm{R}$, but the fraction of structures near the extremes is small. For the trajectories shown in Figure 8, there is bias towards positive twist angles for isomer $\mathrm{A}$, and a more pronounced bias toward negative twist angles for isomer B. As noted previously, these biases have the same net effect but differ in sign because of the $180^{\circ}$ rotation about the $\alpha-\gamma$ axis that leads to this interconversion. The net positive intrinsic rotational strength predicted for both isomers is consistent with the similarity of the heme binding pocket for the two isomers. It must be kept in mind, however, that this similarity may be an artifact resulting from the inadequate starting geometry for isomer B. If a more suitable initial starting geometry can be obtained for isomer $\mathrm{B}$, it is possible that the intrinsic heme rotational strengths for isomer B will be opposite in sign to those for isomer A.

In general, the two orthogonally polarized transitions have intrinsic rotational strengths 
that are opposite in sign, especially at large average twist angles. Thus, the total intrinsic rotational strength is usually small in magnitude compared with the rotational strengths of the individual components. Thus, although the individual components may have $|R| \sim 2 \mathrm{DBM}$ for twist angles of $c a .20^{\circ}$, the net rotational strengths have maximal magnitudes of $\sim 0.5$ DBM. Because of this extensive cancellation and the spread within the individual components, the net intrinsic rotational strength shows no correlation $(r= \pm 0.07)$ with the average twist angle and with ruffling.

The chromophores of the protein matrix, peptide and aromatic side-chain chromophores, influence the Soret $\mathrm{CD}$, and they are included in the calculation of $\mathrm{CD}$ of myoglobin following Tinoco's first-order perturbation approach. The Soret transitions were characterized by the PPP approximation. The peptide and aromatic side-chain chromophores were characterized by the experimental parameters, where available, and semi-empirical MO parameters. The total rotational strength corresponds to the rotational strength of the heme in the protein environment. The total Soret rotational strength in the protein environment shows a weak correlation $(r=$ \pm 0.40 ) with the heme distortions, as shown in Figure 9. This weak correlation is mainly due to the influence of protein environment on heme transitions, since the total intrinsic Soret rotational strength shows no correlation with the heme distortions.

The CD spectra of myoglobin in the Soret region for different MD trajectories were calculated from the rotational strengths assuming Gaussian bands, with a band-width of $14 \mathrm{~nm}$ for the two Soret components. The calculated $C D$ spectra, for different MD trajectories, are compared with experiment in Figure 10. The experimental CD spectrum corresponds mainly to isomer A, which is the predominant form (95\%) in solution, and has a positive Soret band at $\sim 418 \mathrm{~nm}$. The calculated CD spectra of isomer A are in good agreement with the experimental 
curve. The apparent difference between experiment and theory in the wavelength of Soret band is larger due the effect of neglect of iron on transition energies. Extrapolation of the linear relation between the Soret $\mathrm{CD}$ intensity and the isomeric composition of the reconstituted carbonmonoxy myoglobin, suggests a weak negative Soret band for isomer B. ${ }^{14}$ Our calculations, however, predict CD spectra for isomer B that have a positive Soret band. For the crystal-based MD trajectories, ${ }^{9}$ both isomers are predicted to have CD spectra that are similar to the experimental $\mathrm{CD}$ spectrum of isomer $\mathrm{A}$. The NMR-based trajectories ${ }^{10}$ give $\mathrm{CD}$ spectra that are quite different. The MD trajectory with the starting structure of structure \#1 from NMR gives CD spectra with reduced intensities for both isomers, in comparison with those from crystalbased trajectories. The CD spectra of both isomers calculated with the MD trajectory of NMR structure \#8 have positive and negative Soret components, with a reversal of the sign of the couplet between the two isomers.

Our calculations suggest that an important factor in the calculation of Soret CD in myoglobin is the non-planar distortion of the heme. The intrinsic rotational strength of the two Soret components in heme are well correlated with the deviations from planarity and there is a weaker but still significant correlation of the total Soret $\mathrm{CD}$ with these deviations. The nonplanar deviations are a result of the environment of heme in the heme pocket, which is determined by the protein matrix. The starting structure for isomer A in crystal-based MD simulations is the most reliable, and the best agreement with experiment is obtained from these calculations. The heme deformations in isomer $\mathrm{B}$ are similar to those in isomer $\mathrm{A}$, which suggests that the starting structure of isomer $B$, generated by simply rotating the heme in the heme pocket of isomer A, may have been incorrect. The similarity of heme deformations and the protein matrix in these two isomers results in the similar predicted Soret $\mathrm{CD}$ for isomers $\mathrm{A}$ and 
B. The poorer results for NMR-based trajectories are also likely to be the result of inferior starting structures.

\section{CONCLUSIONS}

The protein maintains the symmetry properties of the heme-binding site even when the heme itself is rotated about the $\alpha-\gamma$ axis. The time-averaged macrocycle structures for isomers $\mathrm{A}$ and B largely reflect this site symmetry. This means that isomer A is distorted in a particular way, but when the heme is rotated by $180^{\circ}$, the same distortion is unfavorable in the binding site and the protein causes the macrocycle conformation to change. For example, doming always occurs in the direction of the proximal ligand, although this appears in the NSD results as a positive doming for isomer $\mathrm{A}$ and a negative doming for isomer $\mathrm{B}$.

The 100-ps time averages reveal nanosecond-scale variations in the heme structure, especially for the softest modes of deformation, saddling and ruffling. Even 600-ps MD trajectories are too short for averaging these motions. Nonetheless, starting from different experimentally obtained initial structures and different initial velocities, generally consistent results were obtained. The original heme distortions of the neutron diffraction structure were qualitatively conserved in the MD trajectories, but long-period variations reflect slow, largescale motions of the protein matrix. Examination by NSD of a time series covering an interval of 6.4 ps shows quasiperiodic variations in the out-of-plane deformations, with ruffling having the largest amplitude and longest period, in accord with the normal mode analysis of porphyrins.

The average twist angle shows strong correlation with the ruffling deformation and can be used to characterize the overall heme deformation. The intrinsic rotational strengths of the $x$ - 
and $y$-polarized components of the Soret band correlate well with the ruffling deformation and the average twist angle, but not with other types of deformations. The average twist angle can be used to predict the sign and approximate magnitude of the intrinsic heme rotational strengths.

The calculated Soret CD for the two isomers are qualitatively similar and are similar to the experimental $\mathrm{CD}$ for the equilibrium mixture. The similarity of the $\mathrm{CD}$ of the two isomers reflects the similar heme deformations in the protein matrix of both isomers in the simulations. However, this similarity is likely to be caused by a starting structure for isomer B that resembles too closely that for isomer A.

\section{ACKNOWLEDGEMENTS}

The work was supported by National Institute of Health (Grant number GM-22994) and the Pittsburgh Supercomputer Center (Grant number MCB980029P). Sandia is a multiprogram laboratory operated by Sandia Corporation, a Lockheed Martin Company, for the United States Department of Energy under Contract DE-AC04-94AL85000. 


\section{REFERENCES}

(1) La Mar, G.N.; Toi, H., Krishnamoorthi, R. J. Am. Chem. Soc. 1984, 106, 6395-6401.

(2) Shelnutt, J.A.; Song, X.Z.; Ma, J.G.; Jia, S.L.; Jentzen, W.; Medforth, C.J. Chem. Soc. Rev. 1998, 27, 31-41.

(3) Livingston, D. J.; Davis, N. L.; La Mar, G. N.; Brown, W. D. J. Am. Chem. Soc. 1984, 106, 3025-3026.

(4) Aojula, H. S.; Wilson, M. T.; Morrison, I. E. G. Biochem. J. 1987, 243, 205-210.

(5) Light, W. R.; Rohlfs, R. J.; Palmer, G.; Olson, J. S. J. Biol. Chem. 1987, 262, 46-52.

(6) Neya, K.; Funasaki, N.; Shiro, Y.; lizuka, T.; Imai, K. Biochim. Biophys. Acta 1994, 1208, $31-37$

(7) Chang, C. K.; Ward, B.; Ebina, S. Arch. Biochem. Biophys. 1984, 231, 366-371.

(8) Gersonde, K.; Sick, H.; Overkamp, M.; Smith, K. M.; Parish, D. W. Eur. J. Biochem. 1986, $157,393-404$.

(9) Kiefl, C.; Sreerama, N.; Woody, R.W. Proteins Str. Func. Gen. In preparation.

(10) Kiefl, C.; Sreerama, N.; Woody, R.W. Proteins Str. Func. Gen. In preparation.

(11) Cheng, X.; Schoenborn, B.P. J. Mol. Biol. 1991, 220, 381-399.

(12) Ösapay, K.; Theriault, Y.; Wright, P.E.; Case, D. J. Mol. Biol. 1994, 244, 183-197.

(13) Aojoula, H.S.; Wilson, M.T.; Drake A. Biochem. J. 1986, 237, 613-616.

(14) Aojula, H.S.; Wilson, M.T.; Moore, G.R.; Williamson, D.J. Biochem. J. 1988, 250, 853-858.

(15) Hsu, M.C.; Woody, R.W. J. Am. Chem. Soc. 1971, 93, 3515-3525. 
(16) Caves, L.S.D.; Evanseck, J.D.; Karplus, M. Protein Science 1998, 7, 649-666.

(17) Pariser, R.; Parr, R.G. J. Chem. Phys. 1953; 21, 466-471; 767-776; Pople, J.A. Trans. Faraday Soc. 1953; 49, 1375-1385.

(18) Jentzen, W.; Ma, J.G.; Shelnutt, J.A. Biophys. J. 1998, 74, 753-763.

(19) Jentzen, W.; Song, X.Z.; Shelnutt, J.A. J. Phys. Chem. B 1997, 101, 1684-1699.

(20) Nakamura, M.; Ikeue, T.; Fujii, H.; Yoshimura, T. J. Am. Chem. Soc. 1997, 119, 6284-6291. 
Table 1. Out-of plane displacements (in $\AA$ ) and calculated intrinsic rotational strength (in DBM) for the different 600 -ps timeaveraged structures with the heme in orientation A and B. The trajectories 1-4 are based on the neutron diffraction structure and differ only in their initial velocities. Trajectory 5 has the same initial velocity as trajectory 2 , but has different starting angles of the vinyl groups. The trajectories NMR\#1 and \#8 are based on the NMR solution structures \#1 and \#8 and differ in their starting geometries. Trajectories with the same numbers have the same initial velocities.

\begin{tabular}{|c|c|c|c|c|c|c|c|c|c|c|c|c|c|}
\hline Isomer & & Tot-o ${ }^{a}$ & Tot-s ${ }^{b}$ & error & sad & $r u f$ & dom & $w a v(x)$ & $w a v(y)$ & pro & $\mathrm{R}_{\text {hem-tri }}{ }^{\mathrm{c}}$ & $R_{\text {hem-tr2 }}{ }^{c}$ & $R_{\text {hem-tot }}{ }^{c}$ \\
\hline \multirow[t]{4}{*}{ A } & & 0.428 & 0.426 & 0.007 & 0.098 & 0.371 & 0.020 & -0.020 & 0.111 & 0.146 & 0.785 & -0.448 & 0.337 \\
\hline & & 0.255 & 0.253 & 0.005 & 0.032 & 0.117 & 0.025 & -0.014 & 0.189 & 0.113 & 0.292 & -0.053 & 0.239 \\
\hline & 3 & 0.779 & 0.777 & 0.009 & 0.116 & 0.726 & -0.017 & -0.055 & 0.221 & 0.103 & $1.030^{\circ}$ & -1.031 & -0.001 \\
\hline & Th4 & 0.548 & 0.546 & 0.008 & 0.162 & 0.476 & 0.045 & -0.009 & 0.179 & 0108 & 0.789 & -0.708 & 0.081 \\
\hline \multirow[t]{5}{*}{ B } & & 0.650 & 0.647 & 0.010 & 0.223 & -0.561 & -0.047 & -0.160 & 0.077 & 0.143 & -0.458 & 0.739 & 0.281 \\
\hline & & 0.490 & 0.488 & 0.006 & 0.281 & -0.362 & -0.043 & -0.135 & 0.032 & 0.083 & 0.463 & -0.377 & 0.087 \\
\hline & & 0.262 & 0.261 & 0.004 & 0.123 & -0.120 & -0.062 & -0.141 & 0.012 & 0.120 & 0.096 & 0.226 & 0.322 \\
\hline & 4 & 0.365 & 0.364 & 0.004 & 0.147 & -0.252 & -0.078 & -0.152 & 0.015 & 0.134 & -0.141 & 0.523 & 0.382 \\
\hline & $-T^{\prime} / 5$ & 0.521 & 0.518 & 0.009 & -0.272 & -0.39 & -0.071 & -0.147 & 0.04 & $\theta .1$ & -0.4 & $0 \rightarrow A m$ & 0.2 \\
\hline \multirow[t]{2}{*}{ A } & $5 \mathrm{Na}$ & 0.285 & 0.278 & 0.011 & 0.029 & -0.194 & 0.116 & -0.141 & 0.067 & -0.030 & -0.447 & 0.118 & -0.329 \\
\hline & AMINAS & 0.805 & 0.798 & 0.019 & 0.355 & -0.654 & 0.151 & -0.138 & 0.203 & 0.007 & -0.633 & 0.421 & -0.212 \\
\hline \multirow[t]{2}{*}{ B } & Difiter & 0.263 & 0.235 & 0.020 & 0.098 & -0.043 & -0.088 & -0.053 & 0.182 & -0.013 & 0.069 & -0.009 & 0.061 \\
\hline & NMR\#8 & 0.549 & 0.520 & 0.031 & 0.099 & 0.430 & -0.087 & -0.176 & 0.190 & 0.033 & -0.797 & 0.688 & -0.109 \\
\hline
\end{tabular}


a) tot-0: The total distortion is the root sum of squares of deformations along all (N-3) out-of-plane normal coordinates, or equivalently the root sum of squares of the z-displacements of the 24 atoms of the macrocycle from the mean plane.

b) tot-s: This distortion is the root sum of squares of only the deformations along the six (sad, ruf, dom, wav $(x), w a v(y)$ and $p r o)$ lowest-frequency out-of-plane normal coordinates, i.e., the total distortions of the structure that best simulates the analyzed structure using only these six deformations.

c) The Soret rotational strength $R_{\text {hem-tot }}$ is the sum of the two transitions $R_{\text {hem-trl }}$ and $R_{\text {hem-tr2. }}$. 
Table 2. Correlation coefficients for all discussed structures for isomer A and B.

\begin{tabular}{llll} 
Distortion & $\mathbf{r}_{\text {hem-tr1 }}$ & $\mathbf{r}_{\text {hem-tr2 }}$ & $\mathbf{r}_{\text {hem-tot }}$ \\
\hline Total & 0.22 & 0.09 & 0.43 \\
Saddling & 0.10 & 0.04 & 0.40 \\
Ruffling & 0.11 & 0.15 & 0.11 \\
Doming & 0.11 & 0.19 & 0.22 \\
Waving (x) & 0.22 & 0.11 & 0.35 \\
Waving (y) & 0.22 & 0.20 & 0.12 \\
Propellering & 0.50 & 0.34 & 0.57 \\
\hline Ruffling corr. $^{\mathrm{a}}$ & 0.54 & 0.49 & 0.23 \\
Doming corr. $^{\mathrm{a}}$ & 0.41 & 0.36 & 0.24
\end{tabular}

a) The correction means, the signs of isomer B have been changed. The $180^{\circ}$ flip of the heme changes the sign of $r u f$ to $-r u f$ and of

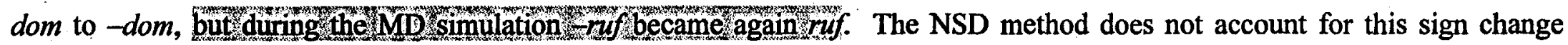
therefore the sign was corrected. 


\section{Figure Legends}

Figure 1. The native (A) and reversed (B) forms which differ by a $180^{\circ}$ rotation of the heme in the protein matrix about the $\alpha-\gamma$ axis. This isomerization exchanges the position of the 2vinyl with the 3-methyl and the 4-vinyl with the 1-methyl group. Both vinyl groups are depicted in the syn-orientation. The pyrrole rings are identified by letters A to D. The view is from the proximal side of the heme.

Figure 2: Illustration of the heme distortions characterized by normal-coordinate structural decomposition of the heme from horse heart cytochrome $c$.

Figure 3: Out-of-plane deformations of the NMR- and crystal-based MD heme structures for isomer A (left) and isomer B (right) for the 600-ps averages. The trajectories T1 to T4 are based on the neutron diffraction structure and differ only in their initial velocities. The trajectories T5 and T6 are based on the NMR solution structures \#1 and \#8, and differ in their starting geometries.

Figure 4: Out-of-plane deformations of the heme structures of (a) T2A and T2B and (b) T6A and T6B for the consecutive 100-ps averages and the 600-ps averages.

Figure 5. Time course of the individual symmetric deformations for an MD simulation for isomer A starting from the neutron-diffraction structure (T2A) for the initial 6.4 ps of the calculation: (a) for sad, ruf, and dom deformations; and (b) for wav(x), wav(y), and pro deformation and the total distortion.

Figure 6. Correlation between the average twist angle $(\tau)$ and ruffling distortion (ruf) for the initial 6.4 ps of T2A. 
Figure 7. Distribution of the twist angles $\tau_{\mathrm{AC}}$ (fill pattern: empty) and $\tau_{\mathrm{BD}}$ (fill pattern: crosshatch) for the crystal- and NMR-based trajectories.

Figure 8. Correlations between average twist angle $(\tau)$ and intrinsic rotational strengths of the two Soret components, separated according to their polarization in the heme-plane, for the crystal-based trajectories of isomers A and B.

Figure 9. Correlations between average twist angle $(\tau)$ and total intrinsic rotational strength for crystal-based trajectories of isomers A and B. 
Figure 1

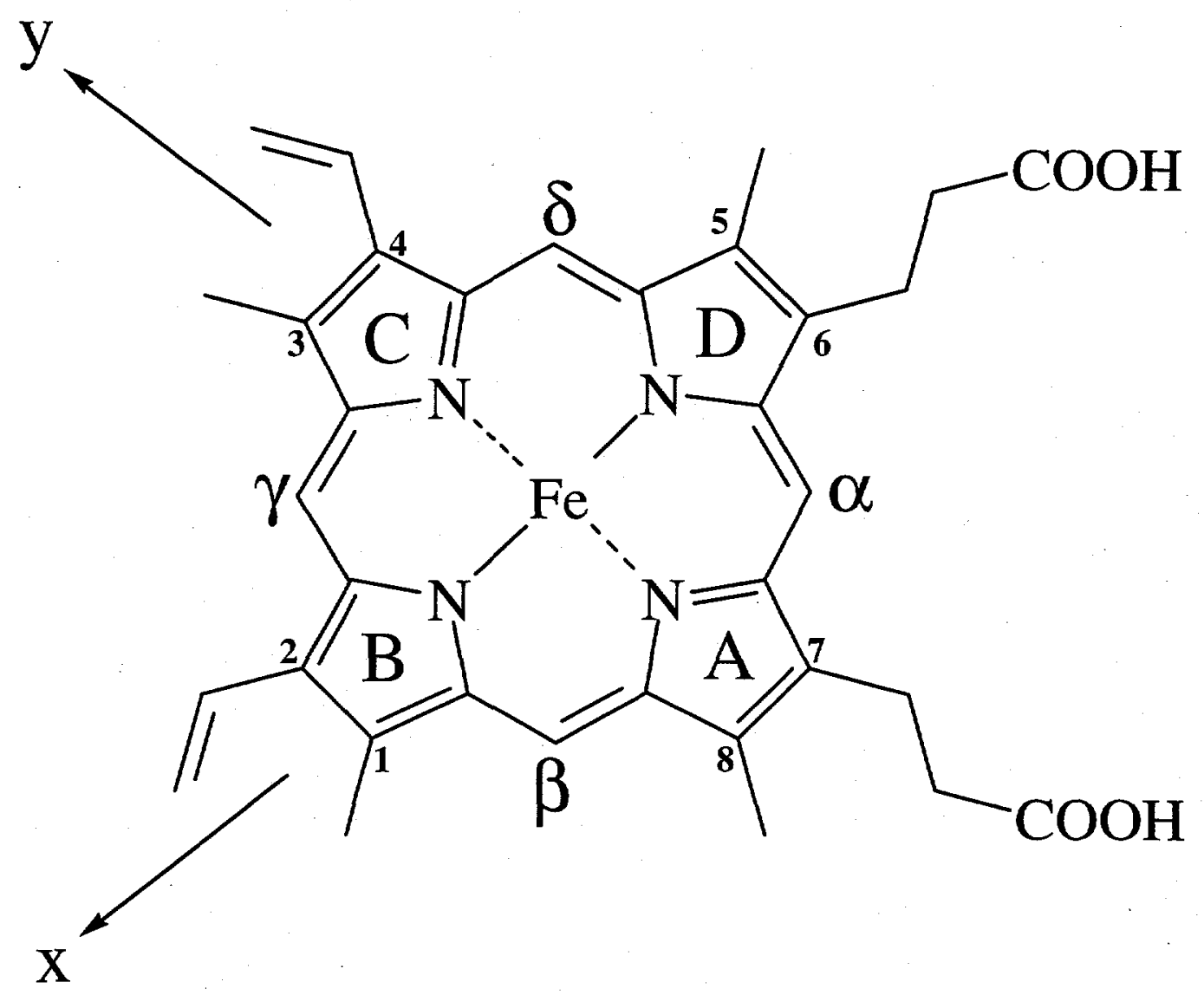


Figure 2
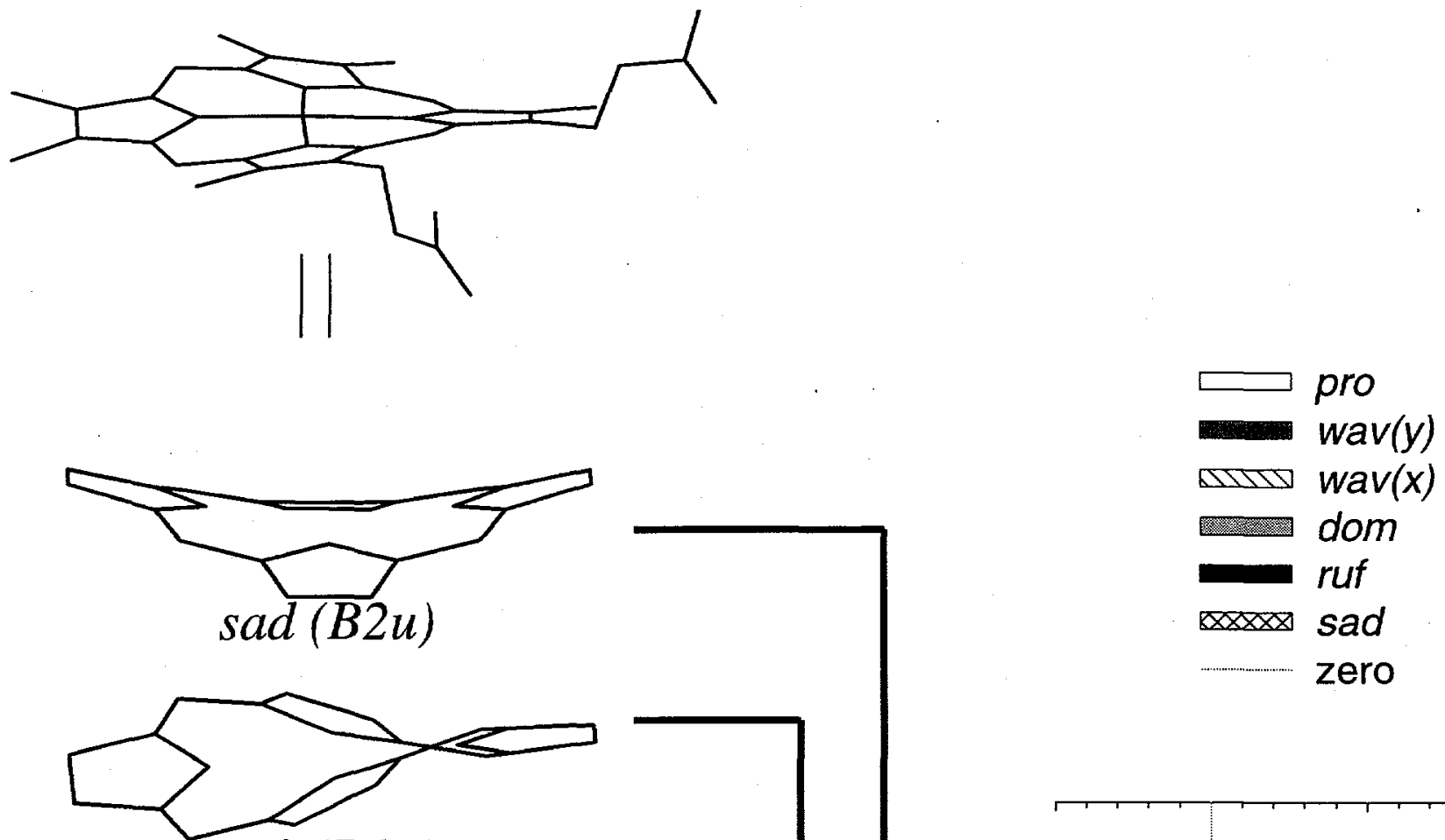

ruf $(B 1 u)$
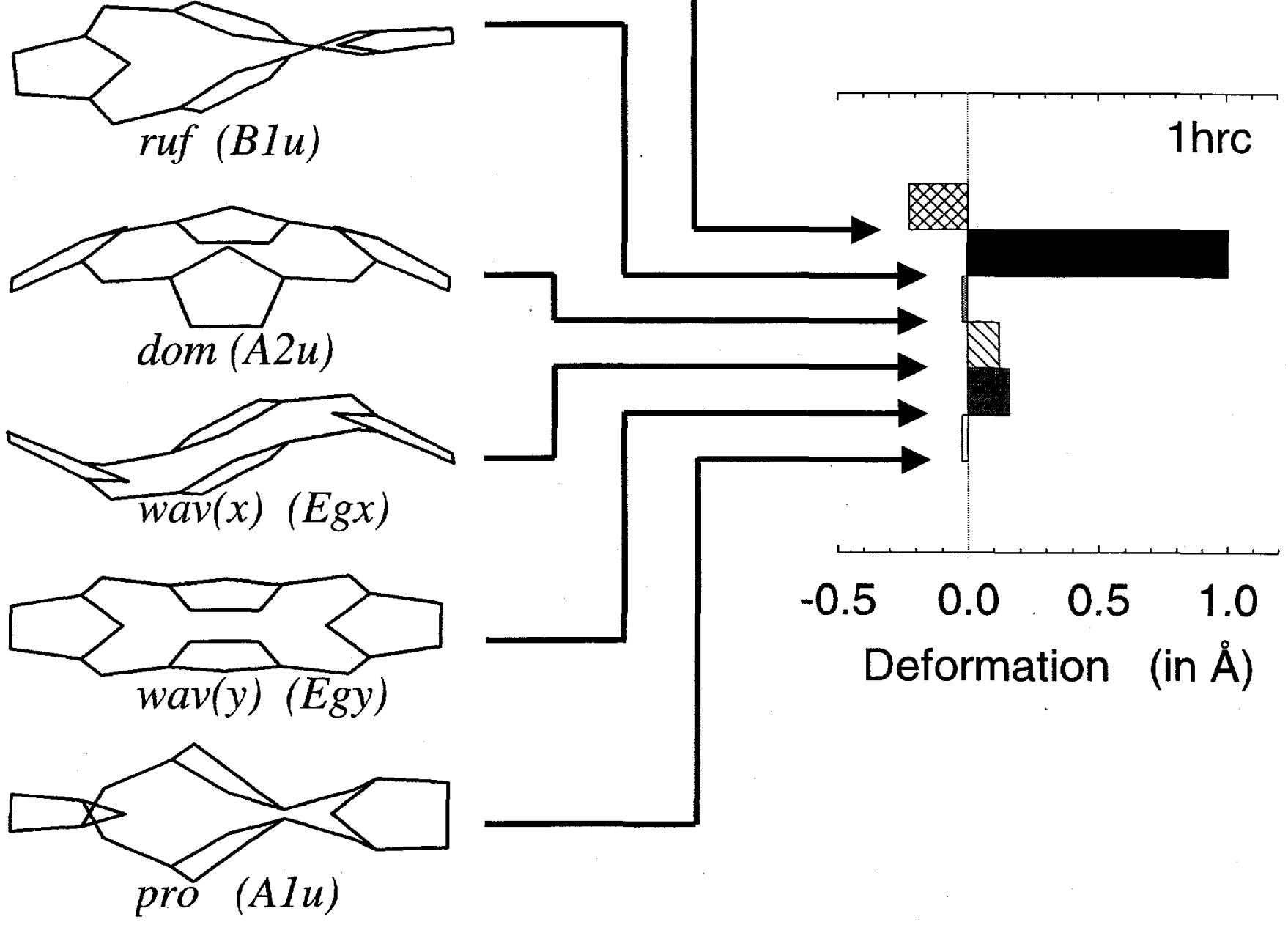
Figure 3
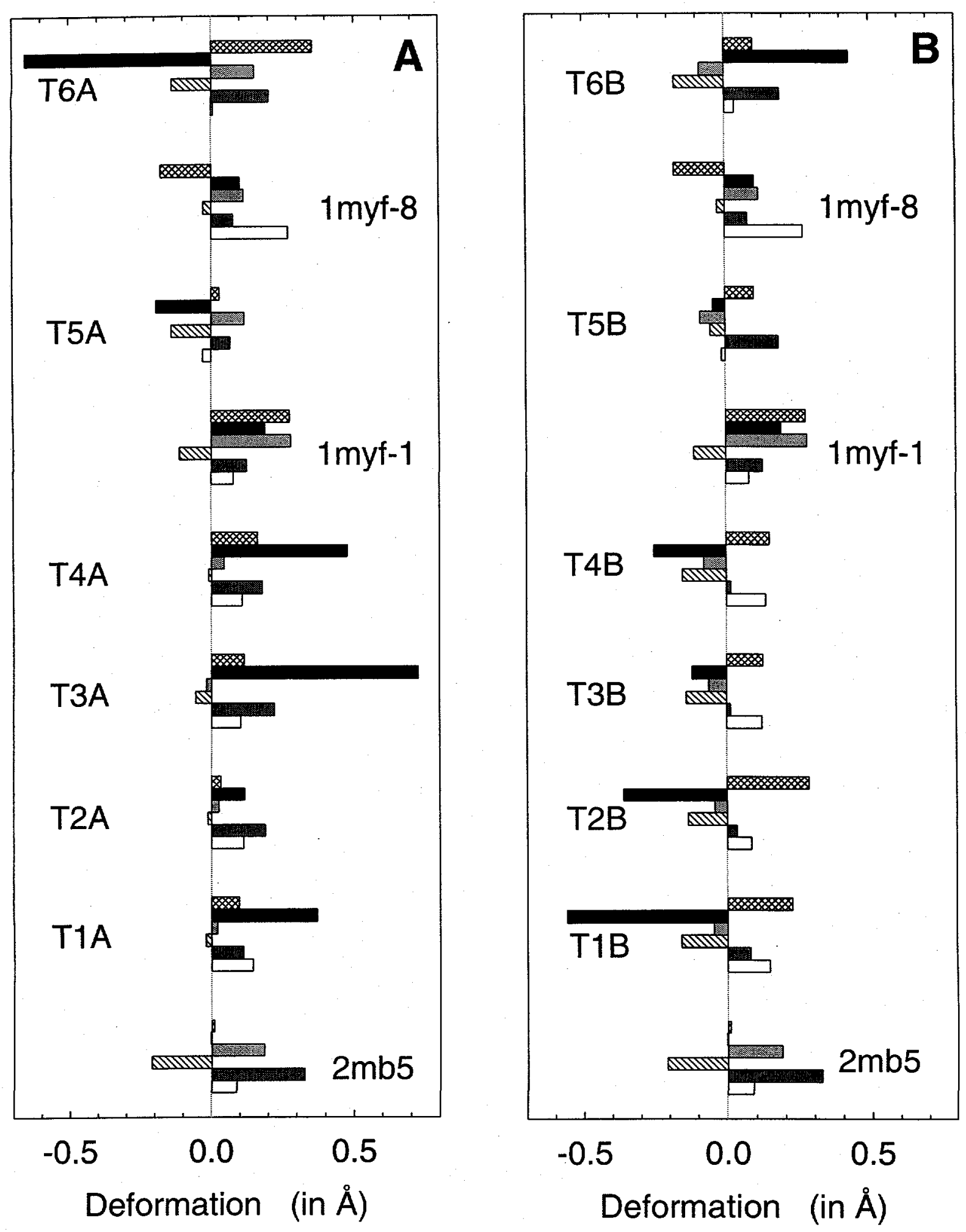
Figure 4(a)
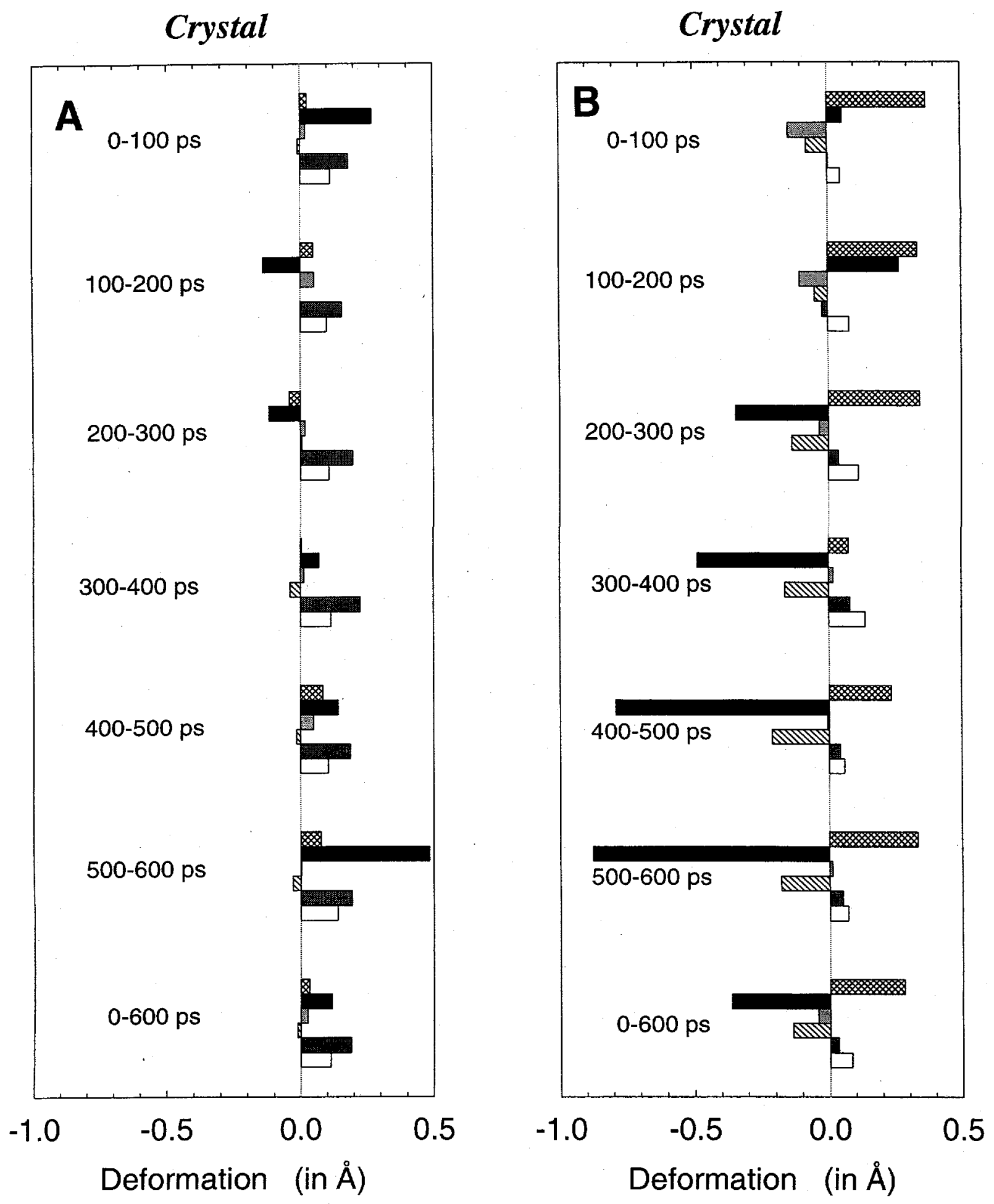
Figure 4(b)
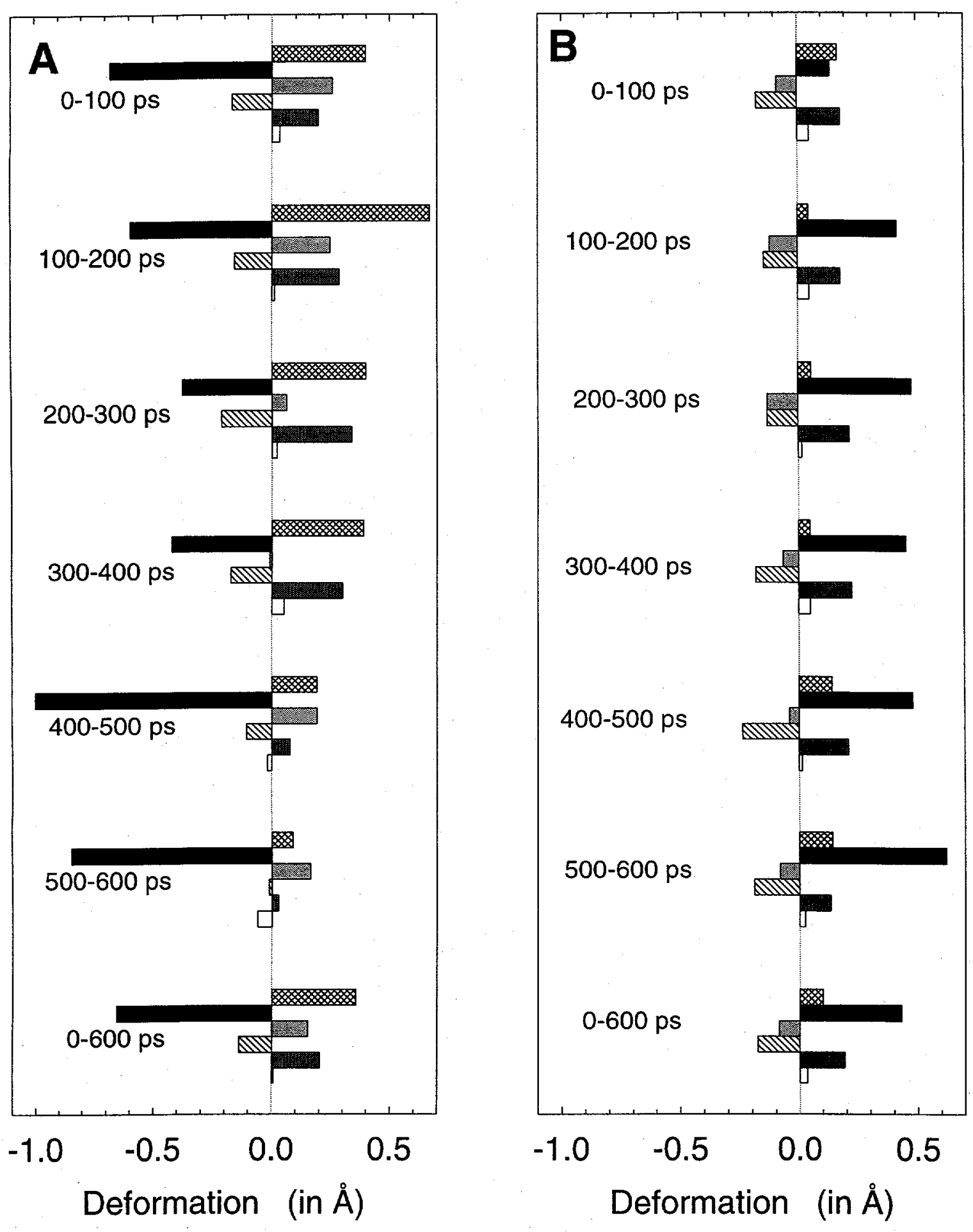
Figure 5
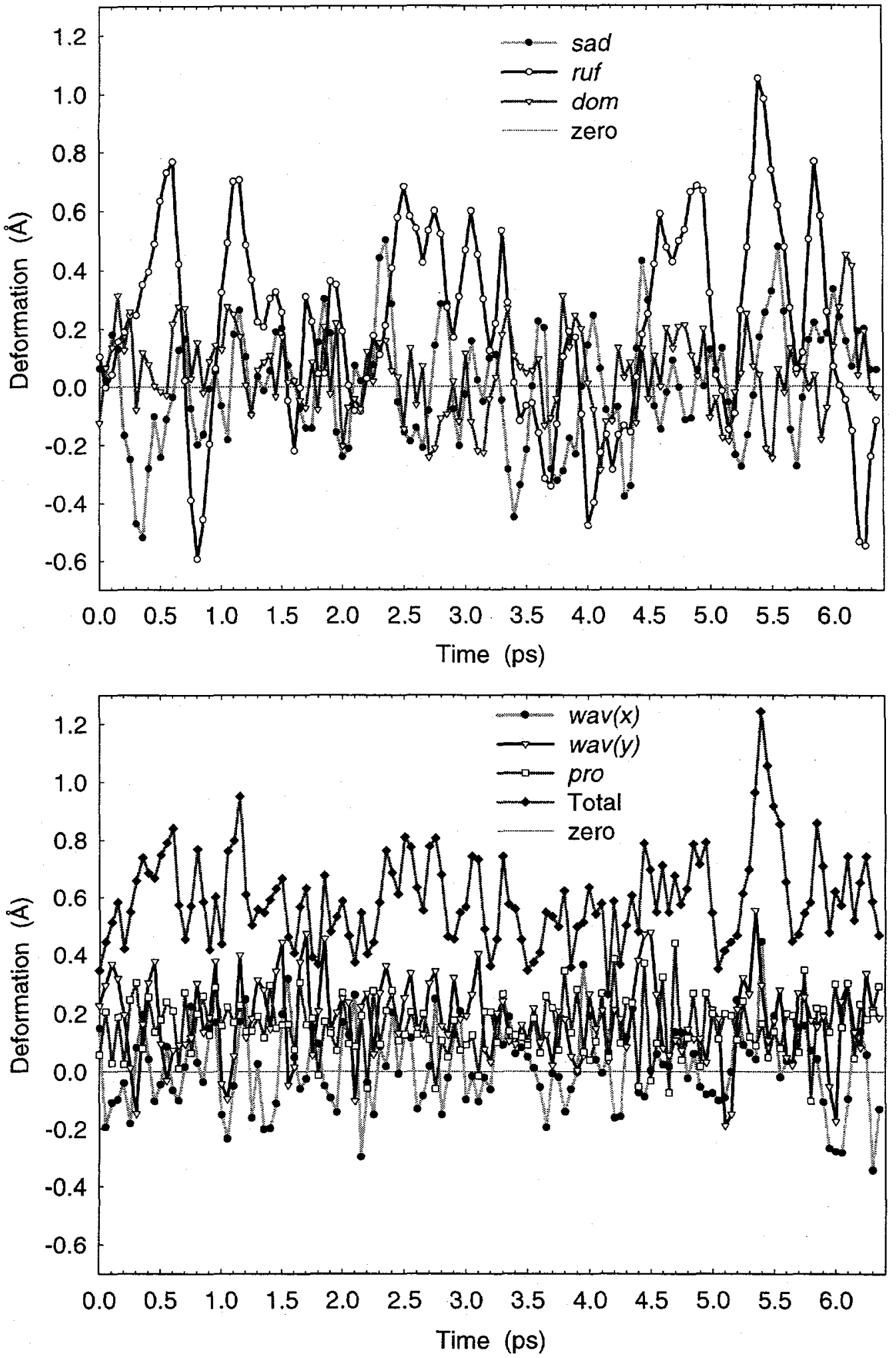
Figure 6

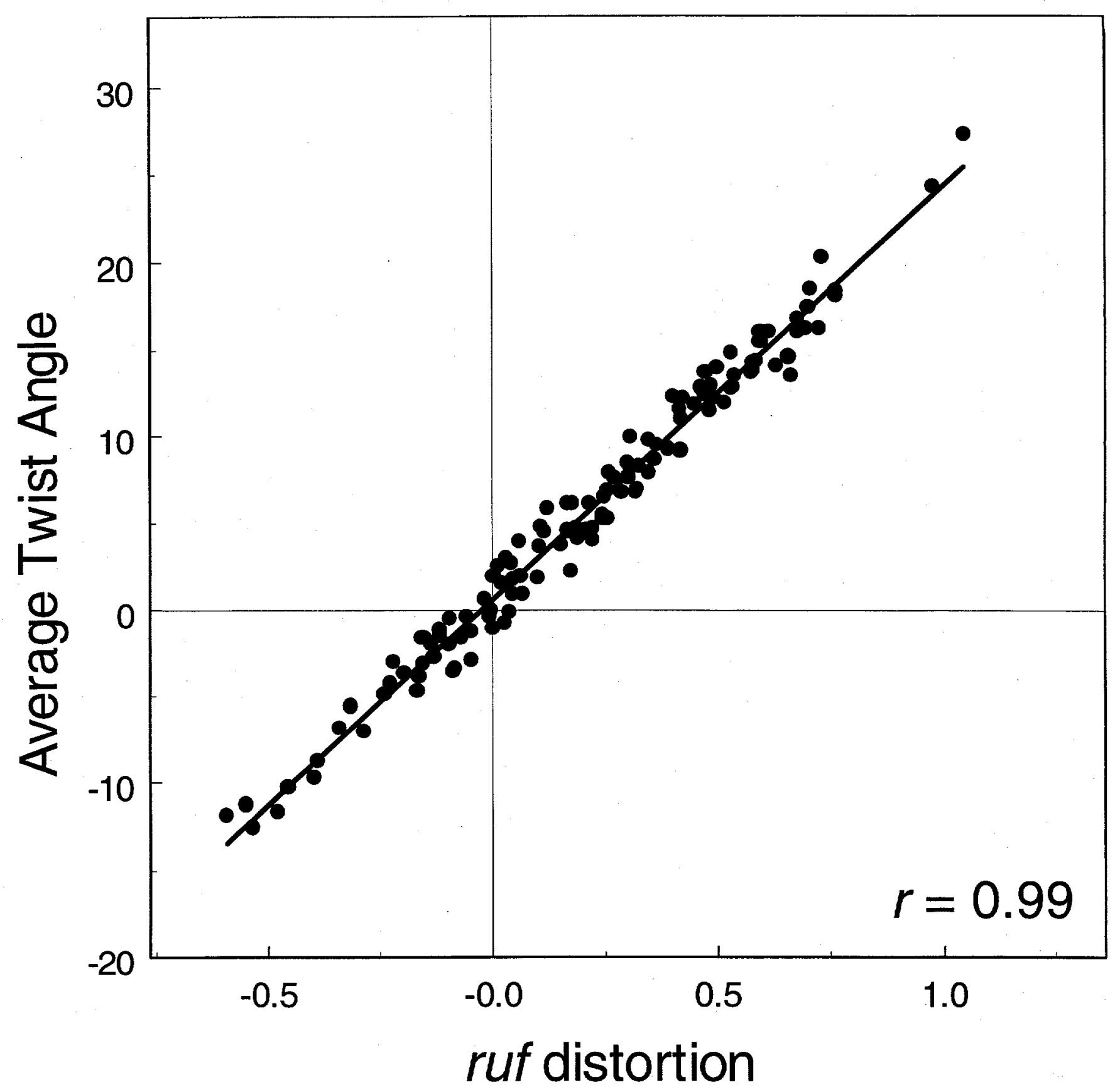


Figure 7

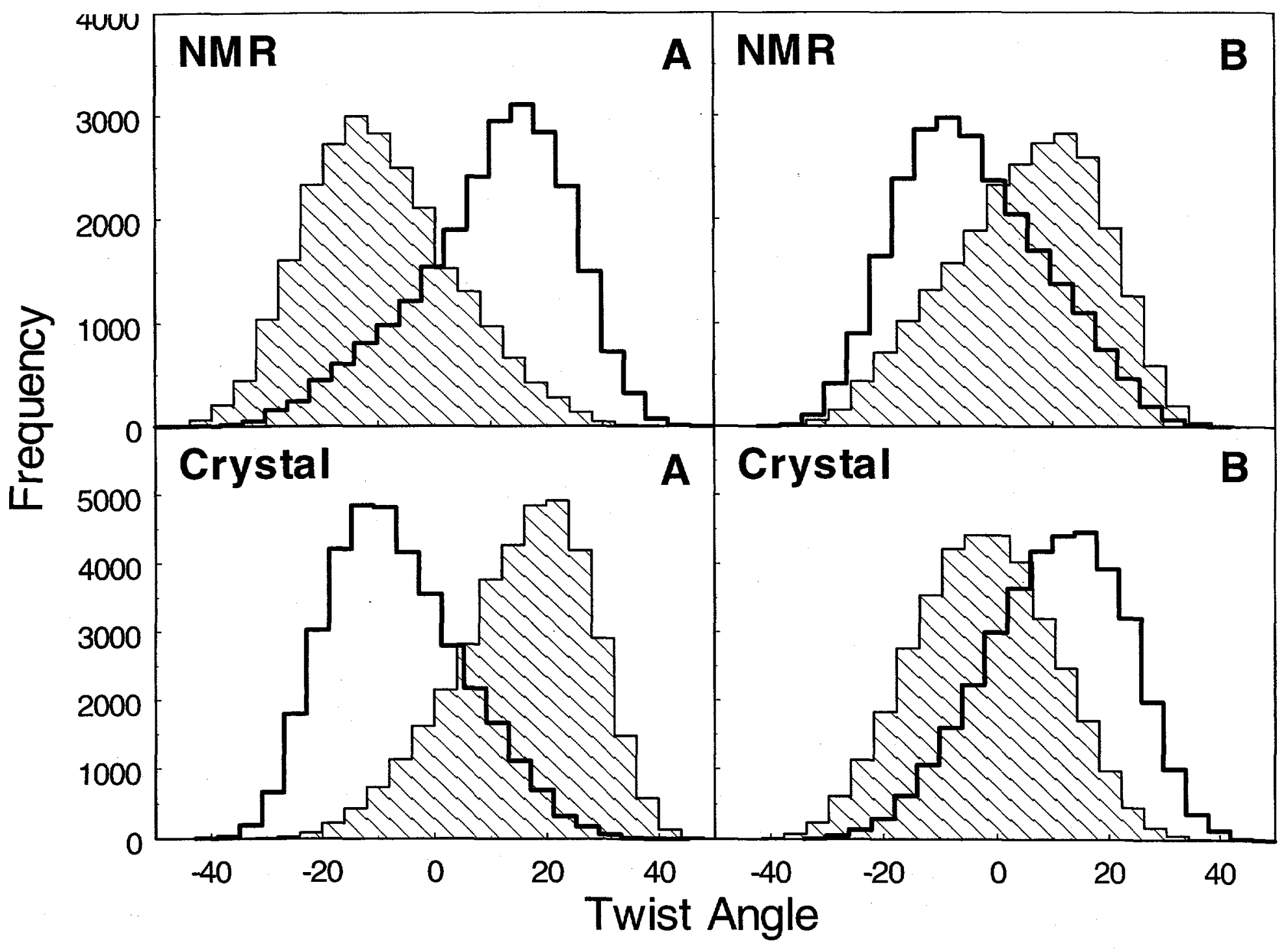


Figure 8

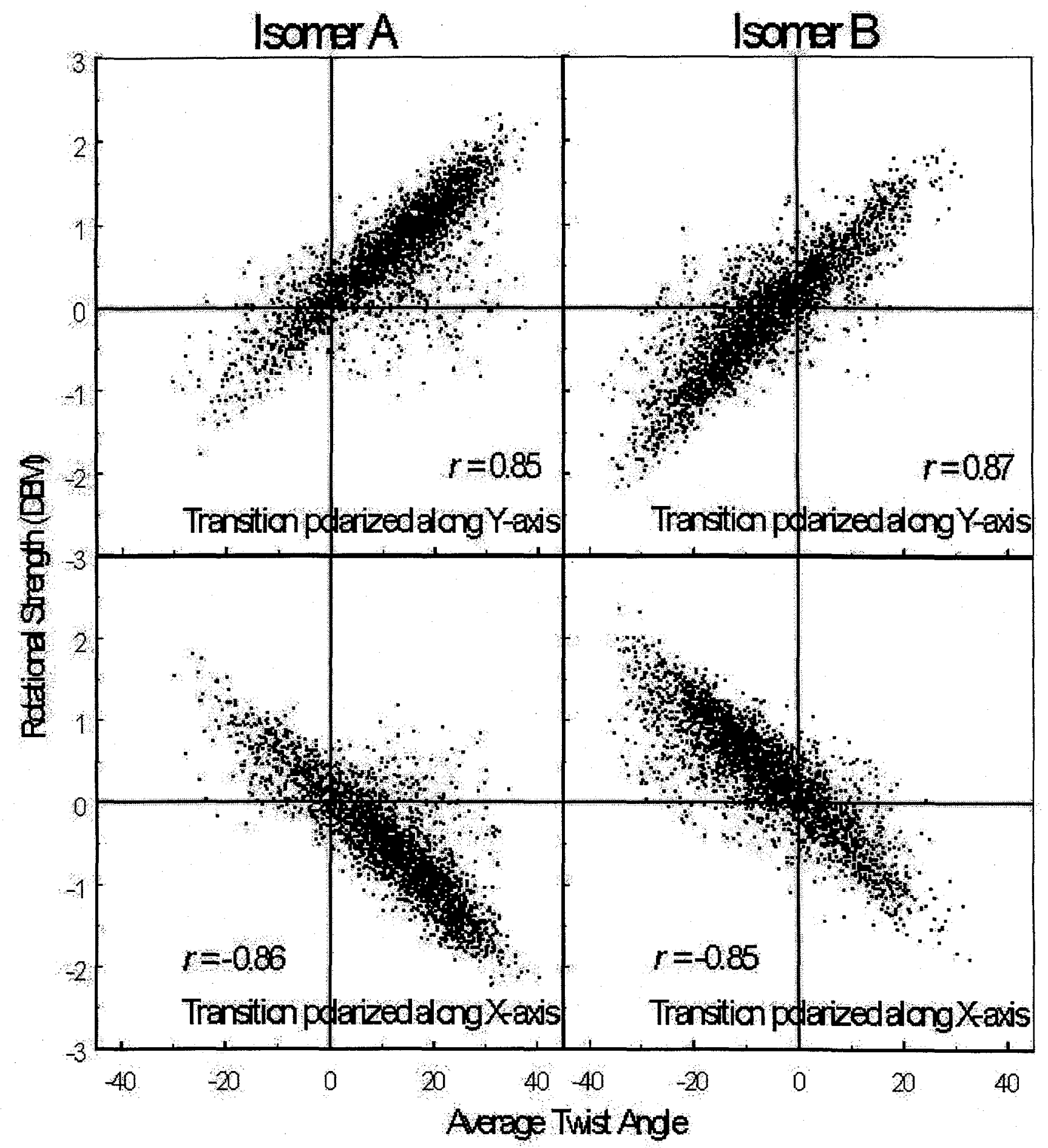


Figure 9

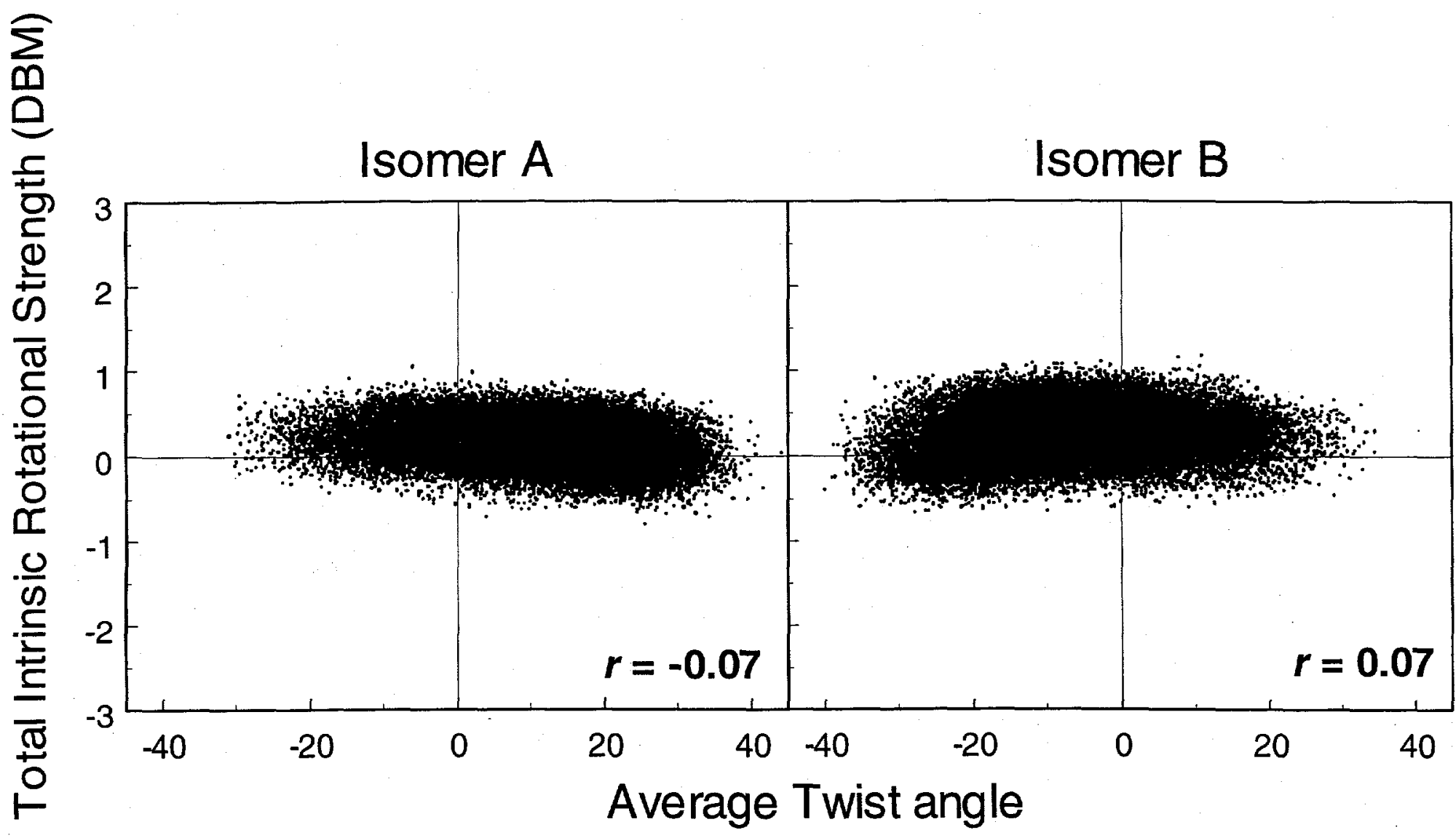

